Published in final edited form as:

Nat Med. ; 18(1): 74-82. doi:10.1038/nm.2577.

\title{
EGFR and MET receptor tyrosine kinase-altered microRNA expression induces tumorigenesis and gefitinib resistance in lung cancers
}

\author{
Michela Garofalo ${ }^{1,9}$, Giulia Romano ${ }^{2,9}$, Gianpiero Di Leva ${ }^{1,9}$, Gerard Nuovo ${ }^{1}$, Young-Jun \\ Jeon ${ }^{1}$, Apollinaire Ngankeu ${ }^{1}$, Jin Sun ${ }^{1}$, Francesca Lovat ${ }^{1}$, Hansjuerg Alder ${ }^{1}$, Gerolama \\ Condorelli ${ }^{3}$, Jeffrey A Engelman ${ }^{4}$, Mayumi Ono ${ }^{5}$, Jin Kyung Rho ${ }^{6}$, Luciano Cascione ${ }^{1,7}$, \\ Stefano Volinia ${ }^{1}$, Kenneth P. Nephew ${ }^{8}$, and Carlo M. Croce ${ }^{1}$ \\ ${ }^{1}$ Department of Molecular Virology, Immunology and Medical Genetics, Comprehensive Cancer \\ Center, Ohio State University, Columbus, OH, USA 43210 \\ ${ }^{2}$ Fondazione IRCCS SDN, 80143, Naples, Italy \\ ${ }^{3}$ Department of Cellular and Molecular Biology and Pathology, IEOS-CNR, Faculty of \\ Biotechnological Science, "Federico II" University of Naples, 80134, Napoli, Italy \\ ${ }^{4}$ Department of Medicine, Harvard Medical School, Boston, MA 02115, USA \\ ${ }^{5}$ Department of Pharmaceutical Oncology, Kyushu University, Fukuoka, Japan \\ ${ }^{6}$ Department of Pulmonary and Critical Care Medicine, University of Ulsan, Seoul, Korea \\ ${ }^{7}$ Department of Clinical and Molecular Biomedicine, University of Catania, Italy \\ ${ }^{8}$ Department of Cellular and Integrated Physiology, Medical Sciences Program, Indiana University \\ School of Medicine and the IU Simon Cancer Center, Bloomington, IN, USA
}

\begin{abstract}
The involvement of the MET oncogene in de novo and acquired resistance of non-small cell lung cancers (NSCLC) to tyrosine kinase inhibitors (TKIs) has been reported, but the precise mechanism by which MET overexpression contributes to TKI-resistant NSCLC remains unclear. MicroRNAs (miRNAs) negatively regulate gene expression and their dysregulation has been implicated in tumorigenesis. To understand the role of microRNAs in TKI-resistant NSCLC, we examined TK receptor-mediated microRNA changes. Here we report that miR-30b/c and miR-221/222, modulated by both EGF and MET receptors, and miR-103, -203, controlled only by MET, play important roles in gefitinib-induced apoptosis and epithelial-mesenchymal transition
\end{abstract}

Users may view, print, copy, download and text and data- mine the content in such documents, for the purposes of academic research, subject always to the full Conditions of use: http://www.nature.com/authors/editorial_policies/license.html\#terms

Correspondence: carlo.croce@osumc.edu.

${ }^{9}$ These authors contributed equally to this work.

Author contributions: M.G. designed research; M.G., R.G., G.D.L, J.J.Y., A.N., F.L., performed research; G.D.L. and J. S. conducted animal experiments; G.N. performed IHC and ISH experiments; H.A. performed microarray experiments and analysis; K.N., G.C., provided discussion and advices; J.A.E., M.O., J.K.R. provided acquired gefitinib-resistant cells, S.V. and L.C. performed bioinformatic analyses; M.G., and C.M.C. wrote the paper.

Competing Financial Interests. The authors declare no competing financial interests. 
(EMT) of NSCLC cells, in vitro and in vivo, by inhibiting the expression of Bim, APAF-1, PKC-E and $S R C$ genes. The finding suggests that modulation of specific microRNAs may provide a therapeutic approach for future treatment of NSCLC.

MicroRNAs repress gene expression by inhibiting mRNA translation or by promoting mRNA degradation and are considered master regulators of different processes, ranging from proliferation ${ }^{1}$ to apoptosis ${ }^{2}$. Altered miRNA expression in various human tumor types has been observed, and critical roles of miRNAs in cancer pathogenesis and response to therapy have been demonstrated ${ }^{3,4}$. Non-small cell lung cancers (NSCLC) account for roughly $85 \%$ of all lung cancer cases ${ }^{5}$. Although NSCLC is a remarkably heterogeneous disease that includes distinct morphological and molecular subtypes, activation of epidermal growth factor receptor (EGFR) and MET (the receptor tyrosine kinase (RTK) for hepatocyte growth factors) is common and associated with RAS/ERK and PI3K/AKT axes stimulation, leading to NSCLC cell proliferation, survival and invasion ${ }^{6}$. Tyrosine kinase inhibitors (TKI) gefitinib and erlotinib effectively target EGFR in NSCLC patients, but these important therapeutic agents are ultimately limited by the emergence of drug resistance mutations and other putative molecular mechanisms. ${ }^{7}$

MET protein expression and phosphorylation have been associated with primary and acquired resistance to EGFR TKI therapy in NSCLC patients ${ }^{8,9}$, strongly implicating MET as an effective therapeutic target to overcome resistance to this important class of drugs in lung cancer ${ }^{10}$. Here we show that EGF and MET receptors, by modulating specific microRNAs, control gefitinib-induced apoptosis and NSCLC tumorigenesis. Our results are the first to identify EGF and MET receptor-regulated microRNAs representing oncogenic signaling networks in NSCLC.

\section{Results}

\section{MicroRNAs modulated by both EGFR and MET}

To identify EGFR- and MET-regulated-miRNAs, we stably silenced EGFR and MET in Calu-1 cells using shRNA lentiviral particles (Fig. 1a) and examined global microRNA expression profiles. In EGFR- and MET-knockdown (EGFR-KD and MET-KD) Calu-1 cells, 35 and 44 significantly dysregulated microRNAs were identified, respectively (Figs. $1 \mathrm{~b}$ and Supplementary Fig. 1a). MicroRNAs with $>1.5$ - (EGFR) and with $>1.7$ - (MET) foldchange are shown. By comparing the two lists, only 8 microRNAs were found to be regulated by both EGFR and MET (Fig. 1c): miR-21 (fold change EGFR-KD $=-1.56$; fold change $\mathrm{MET}_{\mathrm{MED}}=-1.7$ ), $-221 / 222$ (f.c.EGFR-KD $=-1.79 /-1.66$; f.c. $\cdot$ MET-KD $=-2.07 /-1.75$ ), $-30 \mathrm{~b} / \mathrm{c}$ (f.c.EGFR-KD $=-1.81 /-2.4$; f.c.MET-KD $=-3.5 /-4.0$ ), $-29 \mathrm{a} / \mathrm{c}$ (f.c.EGFR-KD $=-1.52 /-1.53$; f.c.MET-KD $=-1.72 /-1.79$ ), and -100 (f.c.EGFR-KD $=-1.55$; f.c.MET-KD= -1.72 ). We initially focused on miR-30b/c and 221/222, downregulated after both MET and EGFR silencing and showing the highest expression level fold-change. We also investigated two microRNAs most differentially induced after MET silencing, miR-103 (f.c.= 2.45) and miR-203 (f.c.= 2.5), based on recent evidence for MET overexpression in de novo and acquired resistance to TKIs ${ }^{8,9}$. Expression levels of these six miRNAs in EGFR-KD and MET-KD Calu-1 cells were validated using qRT-PCR (Supplementary Fig. 1b) and northern blot (Fig. 1d) analysis. 


\section{MiR103, 203, 30b/c and 221/222 target $P K C \varepsilon, S R C, B I M$ and $A P A F 1$}

As MET and EGFR RTKs play a significant role in lung cancer tumorigenesis and progression ${ }^{11}$, we hypothesized that miR-103 and miR-203 (increased after MET knockdown) are tumor suppressors and miR-221/222 and miR-30b/c (decreased after MET and EGFR silencing) are oncogenic. Our search for mRNA targets of miR-103, -203, -221/222, and -30b/c using Targetscan and Pictar computational tools revealed that 3'UTRs of human Apaf-1, BIM, $P k c-\varepsilon$ and $S r c$ genes contain evolutionarily conserved binding sites specific for these miRNAs (Supplementary Fig. 2a). We focused on these genes based on their role in TKI sensitivity (BIM and APAF-1) $)^{12,13}$ and resistance $(S R C)^{14}$ or negative allosteric modulation of EGFR signalling $(P K C-\varepsilon)^{15}$. To investigate whether these miRNAs directly interact with the four putative target genes, we cotransfected pGL3-3'UTR vectors with synthetic miR-103,-203, 221/222 and -30b/c. Decreased luciferase activity indicated direct miR-PKC-E, SRC, APAF-1 and BIM $3^{\prime}$ UTR interactions (Fig. 1e), and target gene repression was rescued by mutations or deletions in the complementary seed sites (Figs. 1e and Supplementary Fig. 2a). Western blot analysis showed an inverse correlation $(p<0.05)$ between miR-221/222, -103, -203, 30b/c expression and target protein amount in a NSCLC cell panel (Supplementary Fig. 2b,c) confirmed by Pearson coefficient (Figs. If and Supplementary Fig. 2d). The immunoblot analysis fully agreed with data obtained using reporter gene assays (Supplementary Results, Figs. 1g-j and Supplementary Fig. 3a,b). Detection of PKC- $\varepsilon$, SRC, APAF-1, BIM proteins in vivo in 110 lung cancer specimens using miRNA in situ hybridization (ISH) followed by immunohistochemistry (IHC) further strengthened the negative correlation between these proteins and miR-103, -203, 221/222 and $-30 \mathrm{~b} / \mathrm{c}$ seen in vitro (Supplementary Table 1). An inverse correlation between miR-203/ SRC, miR-30c/BIM, miR-103/PKC- $\varepsilon$, and miR-222/APAF-1 expression was observed in the majority of the lung cancer tissues (Supplementary Fig. 4a,b). Moreover, MET overexpression was seen in 52\% (57/110) of the same 110 lung tumor samples (demonstrated using miRNA ISH/MET IHC; Supplementary Fig. 5a), low miR-103 and miR-203 expression and high miR-222 and miR-30c expression was observed in METoverexpressing tumors (Fig. 2a; Supplementary Fig. 5a). Interestingly, the majority of MET overexpressing tumors had accompanying metastases (Supplementary Fig. 5b), indicating that MET-regulated microRNAs play a role in metastatic spread of lung cancer cells.

We extended our analysis to 40 independent lung tumors with annotated clinical history (Supplementary Table 2), divided into two groups of "low" and "high" MET and EGFR expression (based on qRT-PCR; Supplementary Fig. 5c). ANOVA confirmed that miR-30b$\mathrm{c}$ and miR-221-222 were differentially expressed between the two groups, whereas Pearson coefficient identified an inverse correlation MET-miR-103, -203 (Fig. 2b,c). The qRT-PCR results were corroborated using IHC analysis for MET and EGFR (Supplementary Fig. 5d). In addition, MET overexpression was observed in tumors that presented distant metastases versus non-metastatic cases and there was no correlation between metastases and EGFR expression in these 40 lung cancers (Fig. 2d and Supplementary Fig. 5e).

\section{MiR-30b-c, 221-222, 103, 203 regulate gefitinib sensitivity}

Having demonstrated that EGFR regulates miR-221-222 and miR-30b-c, we investigated a role for these miRs in gefitinib-induced apoptosis in NSCLC with wild-type EGFR (Calu-1 
and A549 cells) versus EGFR exon 19 deletions (PC9 and HCC827). Calu-1 and A549 cells were completely resistant to all concentrations of gefitinib tested (up to $20 \mu \mathrm{M}$ ); in contrast, PC9 and HCC827 EGFR-mutant cells were significantly growth-inhibited even by low ( 0.1 $\mu \mathrm{M}$ ) dose gefitinib (Fig. 3a), in agreement with a previous study ${ }^{16}$. Interestingly, after gefitinib treatment, marked miR-30b-c and -221-222 downregulation and increased BIM and APAF-1 protein levels were observed, but only in PC9 and HCC827 sensitive cells (Fig. $3 \mathrm{~b}, \mathrm{c})$. Moreover, as previously anticipated ${ }^{16}$, the level of phosphorylated ERKs was markedly decreased in Hcc827 and PC9 but not in Calu-1 cells versus untreated cells (Fig. 3c).

To directly assess the relevance of miR-30b-c and -221-222 in gefitinib-induced apoptosis, we analyzed expression levels of these miRNAs in PC9 GR and HCC827 GR, NSCLC cells with acquired gefitinib resistance, obtained after long-term exposure to increasing drug concentrations $^{8,17}$. In contrast to the gefitinib-responsive parental cells, decreased miR-30b$c$ and -221/222 expression and modulation of their relative targets were not observed (Fig. 3d; Supplementary Fig. 6a). Also of note, miR-30c and miR-221/222 overexpression in gefitinib-sensitive HCC827 and PC9 rendered these cells less drug responsive (Figs. 4a and Supplementary Fig. 7a), and knockdown of miR-30b-c and -221-222 increased gefitinib sensitivity of Calu-1, HCC827GR and PC9GR (Figs. 4a and Supplementary Fig. 7b), indicating that these miRs are important modulators of TKI resistance.

To investigate the contribution of miR-30b-c and 221-222-mediated APAF-1 and BIM downregulation to cellular TKI response, we overexpressed APAF-1 and BIM in A549 resistant cells. Gefitinib-induced PARP cleavage was observed in cells overexpressing BIM and APAF-1 but not in cells transfected with an empty vector plasmid (Fig. 4b). Conversely, response to gefitinib was reduced by BIM and APAF-1 silencing in gefitinib-sensitive HCC827 and PC9 cells (Fig. 4c). We cloned wild type and mutated 3'UTRs of BIM and APAF-1 (used for luciferase assays, Fig. 1e) downstream of BIM and APAF-1 coding sequences and performed caspase $3 / 7$ and viability assays. No increase in cell death was observed after gefitinib treatment of A549 cells cotransfected with wild type $3^{\prime}$ UTRs for BIM and APAF-1 and miR-30b-c and -221-222; conversely, miR binding site mutation or deletion restored the apoptotic response to gefitinib, suggesting that the effect of APAF-1 and BIM on gefitinib sensitivity was directly related to miR-30b-c and -221-222-mediated knockdown of these proteins (Figs. 4d and Supplementary Fig. 7c).

Because miR-30b-c and -221-222 are also regulated by MET, we hypothesized that MET inhibition, by modulating these miRs in common with the EGFR pathway, could overcome gefitinib-resistance in NSCLC. In support of this hypothesis, strong downregulation of miR-30b-c and -221-222 was observed in Calu-1- and A549-MET overexpressing cells (data not shown) treated with SU11274 MET inhibitor or MET knockdown (Supplementary Fig. 8a,b). Moreover, increased caspase 3/7 activity and decreased cell viability were observed in SU11274-treated Calu-1 or Calu-1-MET-KD cells exposed to different gefitinib concentrations. (Supplementary Fig. 8c,d). Taken together, these results suggest that MET inhibition restores gefitinib sensitivity in TKI-resistant Calu-1 cells through the downregulation of miR-30b-c and miR-221-222. 
Other miRNAs commonly modulated by EGFR-MET, including miR-21, miR-29a/c and miR-100 (Fig. 1c) were downregulated in HCC827- and PC9-gefitinib treated cells and found involved in the response to the drug (Supplementary Results, Supplementary Figs. 9, 10 and 11).

Next, we focused on miR-103 and -203, strongly upregulated after MET knockdown in Calu-1 cells (Fig. 1d). As expected, treatment of Calu-1 cells with SU11274 increased $(P<0.05)$ miR-103 and -203 expression (Supplementary Fig. 12a). SRC and PKC- $\varepsilon$ exert pro-survival effects and contribute to gefitinib resistance by activating AKT and ERK signaling pathways ${ }^{14,15}$. Accordingly, miR-103 and -203-overexpression in A549 cells was associated with reduced phosphorylation of AKT and its substrate GSK3 $\beta$ and ERKs (Supplementary Fig. 13a). Engelman et al. previously reported that MET induces gefitinib resistance through persistent PI3K/AKT and ERK signaling activation ${ }^{8}$, and our results indicate that MET overexpression controls gefitinib resistance through activation of the AKT/ERKs pathway, mediated at least in part by miR-103 and -203. In agreement with Engelman et al., enforced expression of miR-103, -203 or Pkc- $\varepsilon$ and Src silencing increased Calu- 1 cell sensitivity to gefitinib (assessed by caspase $3 / 7$ and viability assays, Supplementary Fig. 13b,c). Importantly, we found that miR-103 and -203 decreased and SRC and PKC- $\varepsilon$ expression consequently increased in HCC827 GR cells with acquired MET amplification and gefitinib resistance ${ }^{8}$, corroborating our hypothesis that MET controls the response to TKIs, at least in part through miR-103 and -203 and their respective targets (Supplementary Fig. 13d,e). Finally, to analyze the sensitivity to gefitinib in vivo, we stably transfected A549 cells with GFP-lentivirus constructs containing either full-length miR-103, miR-203 or anti-miR-221 and -30c. MiR-103 and miR-203 overexpression or miR-221 and -30c knockdown resulted in dramatic tumor growth inhibition and increased sensitivity to gefitinib-induced apoptosis in nude mice after two weeks of treatment (Figs. 4e,f and Supplementary Fig. 14a). MiR-221-222 down-regulation and miR-103, -203 upregulation in the xenograft tumors were confirmed by qRT-PCR (Supplementary Fig. $14 b)$.

\section{MiR-103 and -203 reduce NSCLC cell migration and proliferation}

The anti-proliferative activity of miR-103 and -203 in different cancers has been previously reported ${ }^{18,19,20}$. To further investigate the functional role of miR-103 and miR-203 in NSCLC tumorigenesis, we assessed the impact of miR-103 and -203 gain of function or loss of PKC- $\varepsilon$ and SRC on cell migration and cell cycle kinetics. Migration was reduced (by 2fold compared to controls) in cells with increased miR-103 and -203 or decreased $P K C-\varepsilon$ and $S R C$ expression (Fig. 5a). These results were further confirmed by wound-healing assay (Fig. 5b). In addition, A549 and Calu-1 cells transfected with miR-103, -203 or $P k c-\varepsilon$ and $S r c$ siRNAs showed increased G1 cell fraction and a corresponding decreased number of cells in S and G2-M phases, with miR-203 and siSRC having a slightly stronger effect compared to miR-103 and siPKC- $\varepsilon$ (Fig. 5c).

\section{MiR-103, 203 promote mesenchymal-to-epithelial transition}

An association between EMT and development of chemoresistance, including resistance to EGFR targeted therapy, leading to recurrence and metastasis has recently been reported ${ }^{21}$. 
Although identifying the molecular events underlying EMT is an intense area of investigation, what triggers the onset of EMT in tumor cells remains a mystery. We observed a striking change in cellular shape in MET-KD Calu-1 cells from spindle-, fibroblastoid morphology to an epithelial polarized phenotype upon MET-KO (Fig. 6a), prompting us to further investigate whether this morphological change could be due to a mesenchymal-to-epithelial transition. We assessed the expression of key EMT transitionassociated markers and observed decreased expression of mesenchymal markers and increased E-cadherin expression (Fig. 6b,c.d), strongly indicating reversion of Calu-1 cells to an epithelial phenotype after MET knockdown. Importantly, in MET-KD cells, Snail protein expression was lower, localized to the cytoplasm (Fig. 6b) and presumably nonfunctional $^{22}$. It is worth noting that a morphological change in EGFR-KD Calu-1 cells was not observed, and miR-200c $\mathrm{c}^{23,24},-103$ and -203 were not upregulated (Supplementary Fig. 1a). To test if miR-103 and -203 were involved in mesenchymal-epithelial transition, we overexpressed these miRs in Calu-1 cells and observed downregulation of several mesenchymal markers and increased E-cadherin expression, indicating a role for these miRs in the mesenchymal-epithelial transition (Fig. 6e-g; Supplementary Fig. 15a,b). Moreover, $P K C-\varepsilon$ and $S R C$ silencing increased E-cadherin and decreased SNAIL, ZEB1, ZEB2 (zinc finger E-box binding 2), vimentin and fibronectin mRNA levels (Supplementary Fig. 15c). Since it has been recently reported that miR-103 targets Dicer ${ }^{25}$, we investigated the effects of Dicer knockdown on tumorigenesis and gefitinib-induced apoptosis of NSCLC.

Remarkably, almost complete Dicer knockdown reduced not only gefitinib resistance but also migration and the expression of mesenchymal markers of NSCLC cells, suggesting that miR-103 could be involved in the mesenchymal-epithelial transition process also through Dicer downregulation (Supplementary Results and Supplementary Fig. 16). In conclusion, by regulating expression of specific miRs, MET orchestrates the convergence of several EMT-associated pathways, including Dicer, SRC, PKC- $\varepsilon$ and AKT pathways, supporting the possibility that MET targeting could provide a realistic strategy to control EMT and NSCLC progression.

\section{Discussion}

Although initially promising, it is now clear that EGFR inhibitors, including gefitinib and erlotinib, will not cure the majority of NSCLC patients, even in those cancers expressing mutant EGFR. Therefore, a more complete understanding of how these drugs work and the downstream molecular mediators of EGFR will provide critical information to help design strategies to augment their efficacy.

In previous work, we identified MET as a regulator of miR-221-222 expression ${ }^{3}$. To better understand the pathway(s) involved in NSCLC tumorigenesis and drug resistance, we investigated microRNAs modulated by EGFR and MET tyrosine kinases. We focused on miR-30b-c and miR-221-222, regulated by both EGFR and MET, and miR-103, -203, regulated by MET only. We show that gefitinib treatment, by inducing miR-30b-c and 221-222 downregulation and consequent up-regulation of APAF-1 and BIM, previously reported to play a fundamental role in gefitinib-induced apoptosis ${ }^{12}$, triggers programmed cell death in sensitive Hcc827 and PC9 cells but not in resistant Calu-1 and A549 cells. More importantly, downregulation of miR-30b-c and -221-222 was not seen after gefitinib 
treatment in the resistant clones (HCC827 GR and PC9GR) of gefitinib hypersensitive EGFR exon 19 mutant HCC827 and PC9 NSCLC cell lines. We demonstrate that this resistance could be overcome by MET inhibitors, which downregulate miR-30b-c and 221-222 and sensitize NSCLC to gefitinib or by anti-miR-221-222 and anti-30c, which strongly increase gefitinib-sensitivity in xenograft models in vivo.

PTEN loss, by partially uncoupling mutant EGFR from downstream signaling and activating EGFR, contributes to erlotinib resistance ${ }^{26}$. Previously, we found PTEN to be a miR-221-222 $\operatorname{target}^{3}$, making it reasonable to speculate that these two microRNAs play a role in gefitinib-resistance of NSCLC cells, not only through APAF-1 but also through PTEN-regulation. Notably, overexpression of another miR targeting PTEN ${ }^{27,28}$, miR-21, induced gefitinib resistance in HCC827 and PC9 sensitive cells. We also demonstrate that miR-103 and -203, up-regulated after MET silencing or treatment with the MET inhibitor SU11274, induce apoptosis in gefitinib-resistant NSCLC, reduce mesenchymal markers and increase epithelial cell junction proteins, by downregulating expression of PKC- $\varepsilon$, SRC and Dicer.

Remarkably, EMT has recently been shown to play a role in acquired resistance to gefitinib in A549 cells, indicating that mesenchymal status is related to "inherent resistance" to gefitinib or erlotinib in NSCLC ${ }^{29}$. As proposed in the model in Fig. 6h, either MET knockdown or inhibition of MET activity, through up-regulation of miR-103, miR-203, miR-200 $c^{30}$ and the down-regulation of miR-221-222 and miR-30b-c induces gefitinib sensitivity, motility inhibition and mesenchymal-epithelial transition in NSCLC. The identification of prognostic and predictive factors associated with sensitivity or resistance to anti-EGFR agents is a key area of investigation, and aberrant key signaling proteins, including RAS/MEK, AKT/mTOR and MET kinase, have recently been recognized as critical targets ${ }^{8}$. As activation or amplification of MET signaling contributes to TKI resistance through multiple independent mechanisms and leads to rapid evolution of drug resistance, stratifying NSCLCs based on MET expression may allow for individualization of patient tumors. Such a strategy has the potential to increase treatment efficacy by eliminating unnecessary side effects in NSCLC patients who would not benefit from a particular therapeutic regimen.

It has recently been reported that circulating microRNAs in NSCLC patients may represent a noninvasive strategy for predicting drug response, preventing over-diagnosis and -treatment of low-risk disease, allowing for early disease detection in high-risk subjects, and avoiding early metastatic disease ${ }^{31}$. Noninvasive approaches may be particularly significant because not all the persons with lung cancer have operable disease and tumor tissue is not available for genetic analysis. In this regard, in human primary lung tumors, we identified expression of miR-103,-203,-30b-c and -221-222 as prognostic markers. Our data indicate that analysis of miR-103, miR-203, miR-30b-c and miR-221-222 expression levels, and also of miR-21 and $-29 \mathrm{a}, 29 \mathrm{c}, \mathrm{miR}-100$ after initial treatment with TKIs or MET inhibitors, may potentially provide a noninvasive strategy for predicting response to those inhibitors. The possibility of using these miRNAs as serologic markers for lung cancer warrants further study, supported by recent findings identifying miR-221-222 and -21 as potential blood-based biomarkers with diagnostic value in NSCLC patients ${ }^{32,33}$. In addition, our clinical validation studies on 
lung tumor specimens reveal that MET overexpression and consequent absence of miR-103 and -203 may be used to identify primary lung tumors with metastatic capacity. Indeed, reduced expression of miR-103 and -203 could be predictive of more aggressive, early metastatic tumors. Finally, our results using a tumor xenograft model support a novel therapeutic approach based on modulation of these microRNAs by RNA-based therapeutics, which may represent a clinically useful modality to treat TKI resistance in NCSLC patients with or without MET overexpression.

\section{Methods}

\section{TaqMan Array MicroRNA Cards}

For RNA extraction, see the Extended Experimental Procedures. The TaqMan Array Human MicroRNA Card (Applied Biosystem) Set v3.0 is a two card set containing a total of 384 TaqMan MicroRNA Assays per card that enables accurate quantitation of 754 human microRNAs. Included on each array is three TaqMan MicroRNA Assay, endogenous controls to aid in data normalization and one TaqMan MicroRNA Assay not related to human as a negative control. An additional preamplification step is enabled by Megaplex PreAmp Primers, Human Pool Set v3.0 for situations where sensitivity is of the utmost importance or sample is limiting.

\section{In vivo experiments}

Animal studies were performed according to institutional guidelines. A549 cells were stable infected with a control miR, miR-103 and miR-203 or with anti-miR control, anti-miR-221 and anti-30c lentiviruses (SBI). $5 \times 10^{6}$ viable cells were injected s.c. into the right flanks of 6-wk-old male nude mice (Charles River Breeding Laboratories). Treatment started seven days from tumor cell inoculation. Gefitinib was administered Monday through Friday for 2 weeks as oral gavage, at concentrations of $200 \mathrm{mg} / \mathrm{kg}$ in $1 \%$ Tween 80 (Sigma). in sterile milli-Q water (vehicle control was $0.5 \%$ Tween 80 in sterile milli-Q water). Tumor size was assessed twice per week by a digital caliper. The tumor volumes were determined by measuring the length $(l)$ and the width ( $w)$ and calculating the volume $\left(V=l w^{2} / 2\right) .35$ days after injection, mice were sacrificed. Statistical significance between control and treated animals was evaluated by using Student's $t$ test. Animal experiments were conducted after approval of the Institutional animal care and use committee, The Ohio State University.

\section{Migration assay}

Transwell insert chambers with 8- $\mu \mathrm{m}$ porous membrane (Greiner bio-one) were used for the assay. Cells were washed three times with PBS and added to the top chamber in serum-free media. The bottom chamber was filled with media containing $10 \%$ FBS. Cells were incubated for $24 \mathrm{~h}$ at $37^{\circ} \mathrm{C}$ in a $5 \% \mathrm{CO}_{2}$ humidified incubator. To quantify migrating cells, cells on the top chamber were removed by using a cotton-tipped swab, and the migrated cells were fixed in PBS, 25\% glutaraldehyde and stained with Crystal Violet stain, visualized under a phase-contrast microscope, and photographed. Cristal violet-stained cells were moreover solubilized in acetic acid and methanol (1:1) and absorbance was measured at $595 \mathrm{~nm}$. 


\section{Immunofluorescence}

Cells were grown on Labtek II-CC2 Chamber slides (Nunc), fixed with $4 \%$

paraformaldehyde, and permeabilized with $0.2 \%$ Triton X-100/PBS prior blocking with $10 \%$ sheep serum (Caltag Laboratories). All the primary antibodies were from Abcam. Secondary antibodies were goat anti- mouse or -rabbit coupled to Alexa-488 (Invitrogen). F-actin was stained by using phalloidin reagent (Invitrogen). Cell nuclei were visualized with DAPI (Sigma). Slides were mounted with SlowFade Gold antifade reagent (Invitrogen).

\section{Cell death and cell proliferation quantification}

For detection of caspase 3/7 activity, cells were cultured in 96-well plates, in triplicate, treated with gefitinib 5, 10, $15 \mu \mathrm{M}$ and analyzed using Caspase-Glo 3/7 Assay kit (Promega) according to the manufacturer's instructions. Continuous variables are expressed as mean values \pm standard deviation (s.d.). Cell viability was examined with 3-(4,5dimethylthiazol-2-yl)-2,5-dipheniltetrazolium bromide (MTS)-Cell Titer 96 AQueous One Solution Cell Proliferation Assay (Promega), according to the manufacturer's protocol. Metabolically active cells were detected by adding $20 \mu \mathrm{l}$ of MTS to each well. After $1 \mathrm{~h}$ of incubation, the plates were analyzed in a Multilabel Counter (Bio-Rad Laboratories).

\section{Statistical analysis}

Student's t test, one-way analysis of variance (ANOVA) and Fisher's exact test were used to determine significance. All error bars represent the standard error of the mean. Pearson correlation coefficient was calculated to test the inverse relation between miR-103,-203, $-221 / 222,-30 \mathrm{~b} / \mathrm{c}$ and their putative targets and between MET and miR-103, -203. Statistical significance for all the tests, assessed by calculating $p$-value, was $<0.05$.

\section{Supplementary Material}

Refer to Web version on PubMed Central for supplementary material.

\section{Acknowledgments}

We would like to thank K. Huebner and Susan Lutz for revisions to the paper, Paolo Fadda and Sarah Miller for qRT-PCR assistance. We also thank K. Sergott and Ventana Medical Systems for supplying immunohistochemistry reagents used in this study. M. Nuovo kindly provided the photomicroscopy work. We are grateful for research support from The Ohio State University Targeted Investment in Excellence Award, the NIH CA113001 grant and the Kimmel Scholar Award (M.G.).

\section{References}

1. Wu N, et al. MiR-373, a novel regulator of PPP6C, functions as an oncogene in hepatocellular carcinoma. FEBS J. 2011

2. Chan JA, Krichevsky AM, Kosik KS. MicroRNA-21 is an antiapoptotic factor in human glioblastoma cells. Cancer Res. 2005; 65:6029-33. [PubMed: 16024602]

3. Garofalo M, et al. MiR-221\&222 regulate TRAIL resistance and enhance tumorigenicity through PTEN and TIMP3 downregulation. Cancer Cell. 2009; 16:498-509. [PubMed: 19962668]

4. Cochrane DR, Spoleastra Howe EN, Nordeen SK, Richer JK. MicroRNA-200c mitigates invasiveness and restores sensitivity to microtubule-targeting chemotherapeutic agents. Mol Cancer Ther. 2009; 8:1055-66. [PubMed: 19435871] 
5. Molina JR, Yang P, Cassivi SD, Schild SE, Adjei AA. Non-small cell lung cancer: epidemiology, risk factors, treatment, and survivorship. Mayo Clin Proc. 2008; 83:584-94. [PubMed: 18452692]

6. Sekido Y. Genomic abnormalities and signal transduction dysregulation in malignant mesothelioma cells. Cancer Sci. 2010; 101:1-6. [PubMed: 19793348]

7. Politi K, Fan PD, Shen R, Zakowski M, Varmus H. Erlotinib resistance in mouse models of epidermal growth factor receptor-induced lung adenocarcinoma. Dis Model Mech. 2010; 3:111-9. [PubMed: 20007486]

8. Engelman JA, et al. MET amplification leads to gefitinib resistance in lung cancer by activating ERBB3 signaling. Science. 2007; 316:1039-43. [PubMed: 17463250]

9. Benedettini E, et al. Met activation in non-small cell lung cancer is associated with de novo resistance to EGFR inhibitors and the development of brain metastasis. Am J Pathol. 2010; 177:415-23. [PubMed: 20489150]

10. Agarwal S, et al. Association of constitutively activated hepatocyte growth factor receptor (Met) with resistance to a dual EGFR/Her2 inhibitor in non-small-cell lung cancer cells. Br J Cancer. 2009; 100:941-9. [PubMed: 19240716]

11. Stabile LP, et al. Targeting of both the c-Met and EGFR pathways results in additive inhibition of lung tumorigenesis in transgenic mice. Cancers (Basel). 2010; 2:2153-2170. [PubMed: 21390244]

12. Cragg MS, Kuroda J, Puthalakath H, Huang DC, Strasser A. Gefitinib-induced killing of NSCLC cell lines expressing mutant EGFR requires BIM and can be enhanced by BH3 mimetics. PLoS Med. 2007; 4:1681-89. [PubMed: 17973573]

13. Deng WG, Knon J, Ekmekcioglu S, Poindexter NJ, Grimm EA. IL-24 gene transfer sensitizes melanoma cells to erlotinib through modulation of the APAF-1 and Akt signaling pathways. Melanoma Res. 2010

14. Qin B, et al. Activated Src and Ras induce gefitinib resistance by activation of signaling pathways downstream of epidermal growth factor receptor in human gallbladder adenocarcinoma cells. Cancer Chemother Pharmacol. 2006; 58:577-84. [PubMed: 16532343]

15. Weisheit S, Schafer C, Mertens C, Berndt A, Liebmann C. PKC $\varepsilon$ acts as negative allosteric modulator of EGF receptor signaling. Cell Signal. 2011; 23:436-48. [PubMed: 20965248]

16. Turke AB, et al. Preexistence and clonal selection of MET amplification in EGFR mutant NSCLC. Cancer Cell. 2010; 17:77-88. [PubMed: 20129249]

17. Yoshida T, et al. Effects of Src inhibitors on cell growth and epidermal growth factor receptor and MET signaling in gefitinib-resistant non-small cell lung cancer cells with acquired MET amplification. Cancer Sci. 2010; 101:167-72. [PubMed: 19804422]

18. Lee KH, et al. Epigenetic silencing of microRNA miR-107 regulates cyclin-dependent kinase 6 expression in pancreatic cancer. Pancreatology. 2009; 9:293-301. [PubMed: 19407485]

19. Viticchie' G, et al. MiR-203 controls proliferation, migration and invasive potential of prostate cancer cell lines. Cell Cycle. 2011; 10:1121-31. [PubMed: 21368580]

20. Yuan Y, et al. MicroRNA-203 inhibits cell proliferation by repressing $\delta$ Np63 expression in human esophageal squamous cell carcinoma. BMC Cancer. 2011; 7:11-57.

21. Sabbah M, et al. Molecular signature and therapeutic perspective of the epithelial-to-mesenchymal transitions in epithelial cancers. Drug Resist Updat. 2008; 11:123-51. [PubMed: 18718806]

22. Wu Y, Zhou BP. Snail: More than EMT. Cell Adh Migr. 2010; 4:199-203. [PubMed: 20168078]

23. Wellner U, et al. The EMT-activator ZEB1 promotes tumorigenicity by repressing stemnessinhibiting microRNAs. Nat Cell Biol. 2009; 11:1487-95. [PubMed: 19935649]

24. Chang CJ, et al. p53 regulates epithelial-mesenchymal transition and stem cell properties through modulating miRNAs. Nat Cell Biol. 2011; 13:317-23. [PubMed: 21336307]

25. Martello G, et al. A MicroRNA targeting dicer for metastasis control. Cell. 2010; 141:1195-207. [PubMed: 20603000]

26. Sos ML, et al. PTEN loss contributes to erlotinib resistance in EGFR-mutant lung cancer by activation of Akt and EGFR. Cancer Res. 2009; 15:3256-61. [PubMed: 19351834]

27. Meng F, et al. MicroRNA-21 regulates expression of the PTEN tumor suppressor gene in human hepatocellular cancer. Gastroenterology. 2007; 133:647-58. [PubMed: 17681183] 
28. Zhang JG, et al. MicroRNA-21 (miR-21) represses tumor suppressor PTEN and promotes growth and invasion in non-small cell lung cancer (NSCLC). Clin Chim Acta. 2010; 411:846-52. [PubMed: 20223231]

29. Rho JK, et al. Epithelial to mesenchymal transition derived from repeated exposure to gefitinib determines the sensitivity to EGFR inhibitors in A549, a non-small cell lung cancer cell line. Lung Cancer. 2009; 63:219-26. [PubMed: 18599154]

30. Ceppi $\mathrm{P}$, et al. Loss of miR-200c expression induces an aggressive, invasive, and chemoresistant phenotype in non-small cell lung cancer. Mol Cancer Res. 2010; 8:1207-16. [PubMed: 20696752]

31. Gao W, Liu L, Lu X, Shu Y. Circulating microRNAs: possible prediction biomarkers for personalized therapy of non-small-cell lung carcinoma. Clin Lung Cancer. 2011; 12:14-7. [PubMed: 21273174]

32. Boeri M, et al. MicroRNA signatures in tissues and plasma predict development and prognosis of computed tomography detected lung cancer. Proc Natl Acad Sci U S A. 2011; 108:3713-8. [PubMed: 21300873]

33. Wei J, et al. Identification of plasma microRNA-21 as a biomarker for early detection and chemosensitivity of non-small cell lung cancer. Chin J Cancer. 2011; 30:407-14. [PubMed: 21627863] 

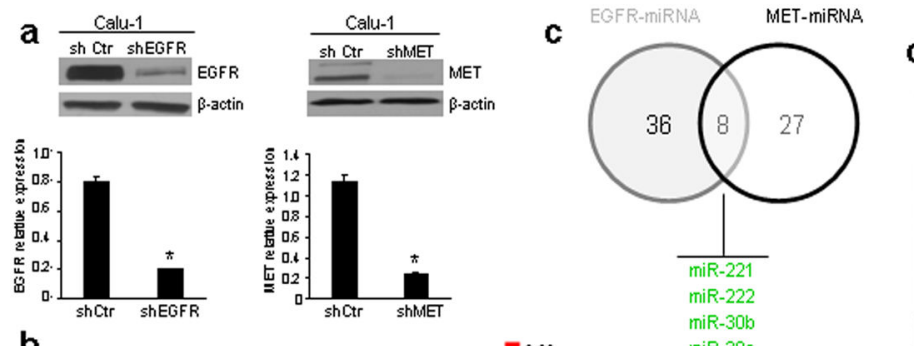

b
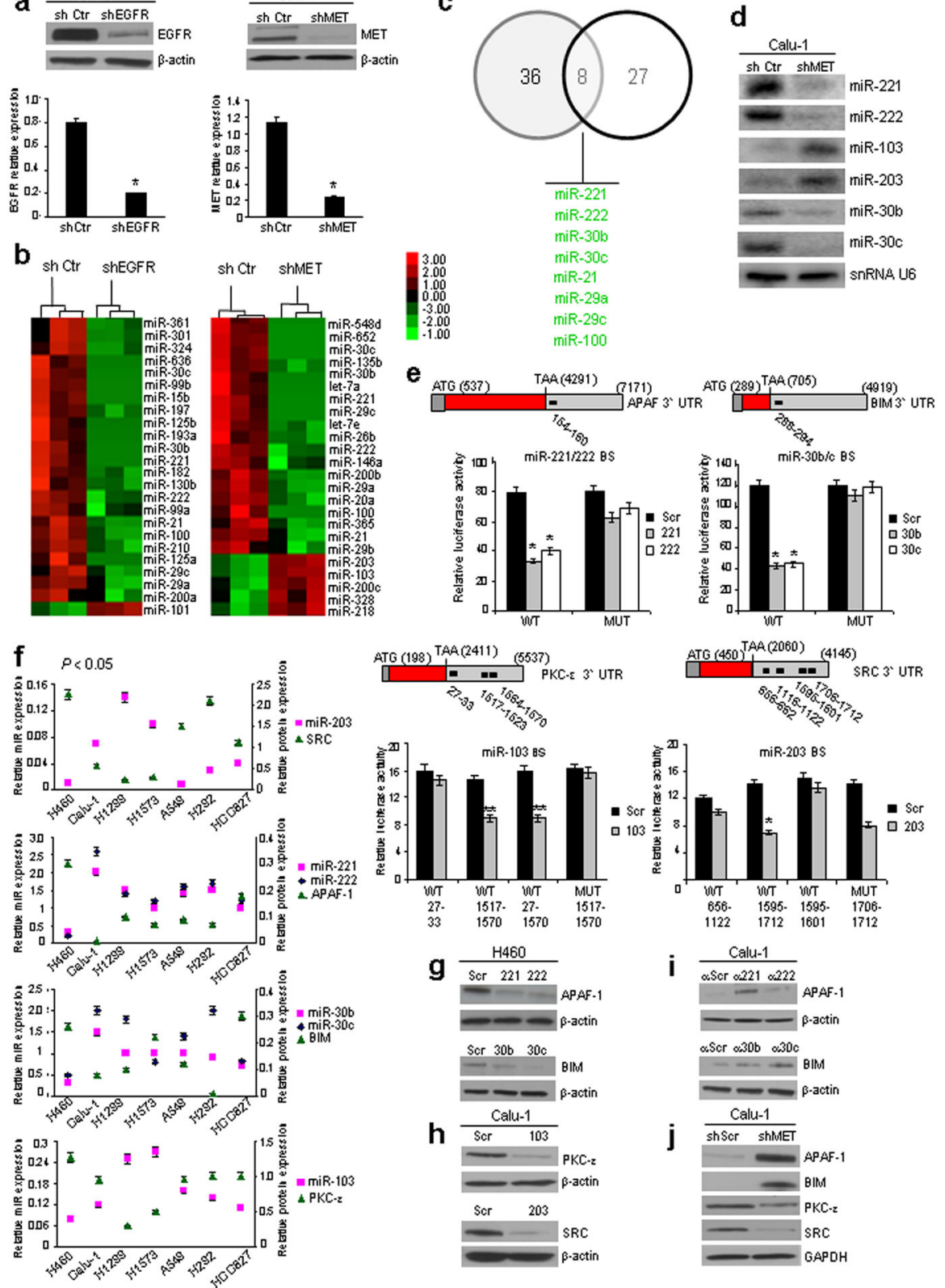

Figure 1. MiR-221-222, 30b-c, 103, 203 target APAF-1, BIM, PKC- $\varepsilon$ and SRC

(a) EGFR and MET proteins and mRNAs down-regulation after EGFR and MET silencing. (b) Unsupervised hierarchical clustering based on miRNA expression profiles in shControl versus shEGFR and shMET-Calu- 1 cells at a $p$ value $<0.05$. (c) Intersection of shEGFR and shMET regulated microRNAs. (d) Northern blots showing miR-221,-222, 103, -203, -30b, and -30c deregulation after MET knockdown. SnRNA U6, loading control. (e) Decreased luciferase activity indicated direct miR-PKC- $\varepsilon, S R C, A P A F-1$ and $B I M 3^{\prime}$ UTR interactions (Fig. 1e) and target gene repression was rescued by mutations or deletions in the complementary seed sites. In the case of SRC only the site 1595-1601 is implicated in the binding with miR-203; deletion of the site 1706-1712 did not rescue luciferase activity (See also Supplementary Fig. 2). Relative repression of firefly luciferase expression was 
standardized to a transfection control. (f) Inverse correlation between miR-103,-203, 221-222 and -30b-c and target proteins in a panel of NSCLC cells. Correlation coefficients of -0.91 (miR-203/SRC), -0.92 (miR-221/APAF-1), -0.90 (miR-222/APAF-1), -0.55 (miR-30b/BIM), -0.91 (miR-30c/BIM), -0.87 (miR-103/PKC- $)$ ), $P<0.05$. (g) MiR-221\&222, miR-30b-c overexpression decreased endogenous levels of APAF-1 and BIM. (h) MiR-103 and miR-203 overexpression decreased endogenous levels of PKC- $\varepsilon$ and SRC. (i) AntimiR-221-222 and anti-miR-30b-c increased APAF-1 and BIM expression. (j) MET knockdown induced APAF-1 and BIM upregulation and SRC and PKC- $\varepsilon$ down-regulation. Results are representative of at least, three independent experiments. Error bars depict \pm s.d. $* P<0.001$, ** $P<0.05$ by two tailed student's $t$ test. 


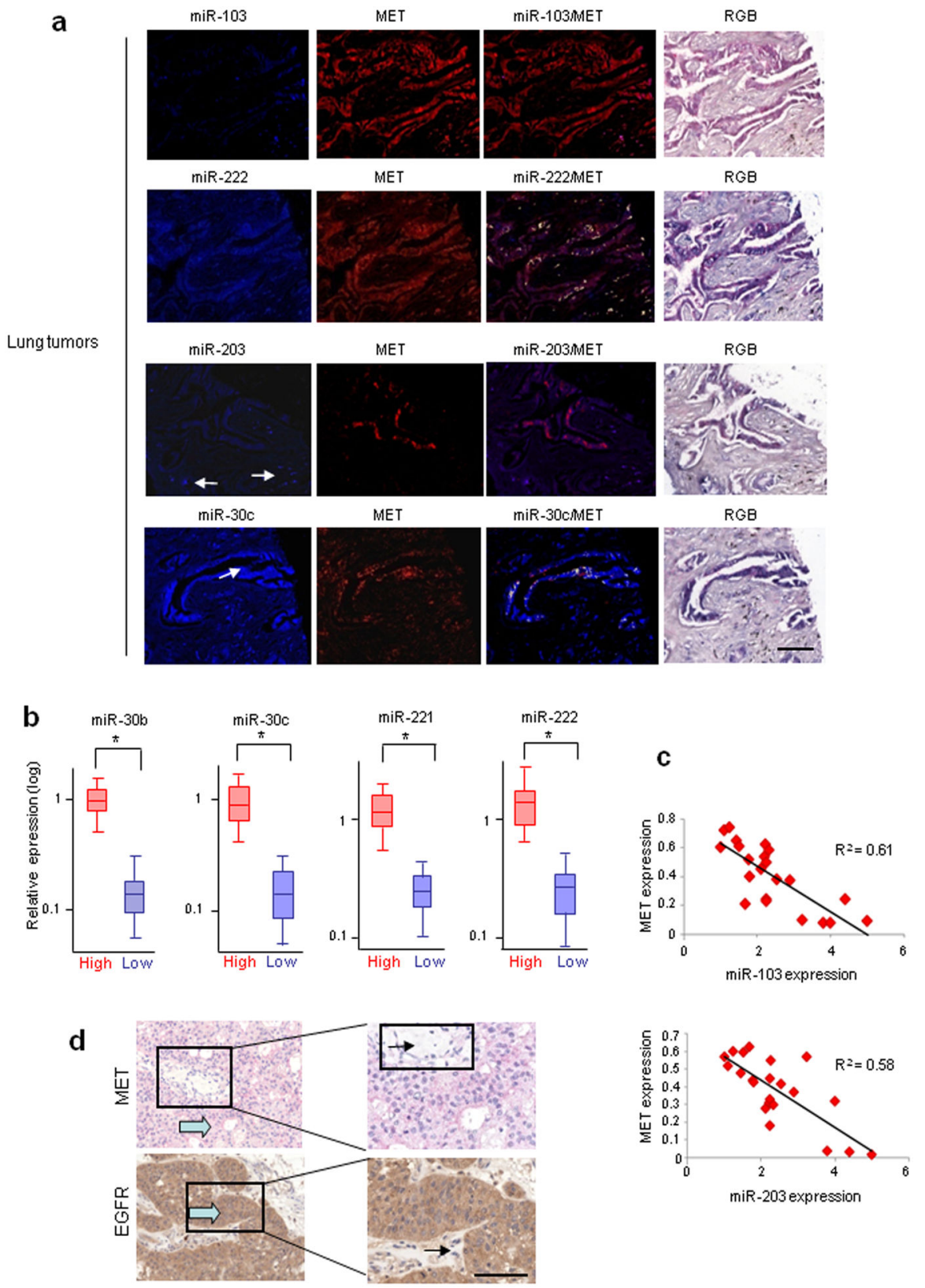

Figure 2. MiR-103-, 222-, 203-, 30c-MET co-expression analyses

(a) 110 lung cancer tissues were analyzed for miR-103, -222, -203, -30c expression by ISH and then for MET by IHC. Upper row, from the left miR-103 signal (blue), MET (red) and the mixed signal in which fluorescent yellow is indicative of miR and protein co-expression; note the lack of miR-103 in presence of MET expression. In the serial section of the same cancer (row below) one sees miR-222, the MET image and the co-expression of miR-222 and MET. Many cancer cells positive for miR-222 also express MET (yellow). The arrows in the left panel (third row) depict benign stromal cells that express miR-203 (blue) and do not express MET. Next panels represent MET (red) and the mixed signal. The arrow in the fourth row depicts cancer cells positive for miR-30c; next panels depict MET signal and the co-expression of miR-30c (yellow). Right panels show the RGB image of the ISH/lHC 
reaction. (b) Box plots showing miR-30b-c, -221-222 expression in 40 lung cancer patients. Real time PCR was used to classify tumors into two groups: EGFR-MET low and EGFR-

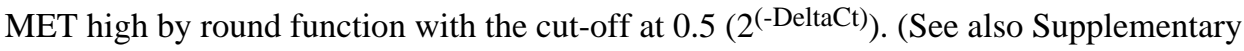
Fig. 5). ${ }^{*} P<0.0001$ by Student's t test. (c) XY Scatter plots showing inverse correlation between MET-103, MET-203. $P<0.0001$. (d) MET and EGFR immunostaining on 40 lung tumor tissues. One representative case of 17 metastatic tumors expressing both MET and EGFR is shown. Large arrow $=$ tumor cells, small arrow $=$ stroma. Scale bar, $100 \mu \mathrm{m}$. 


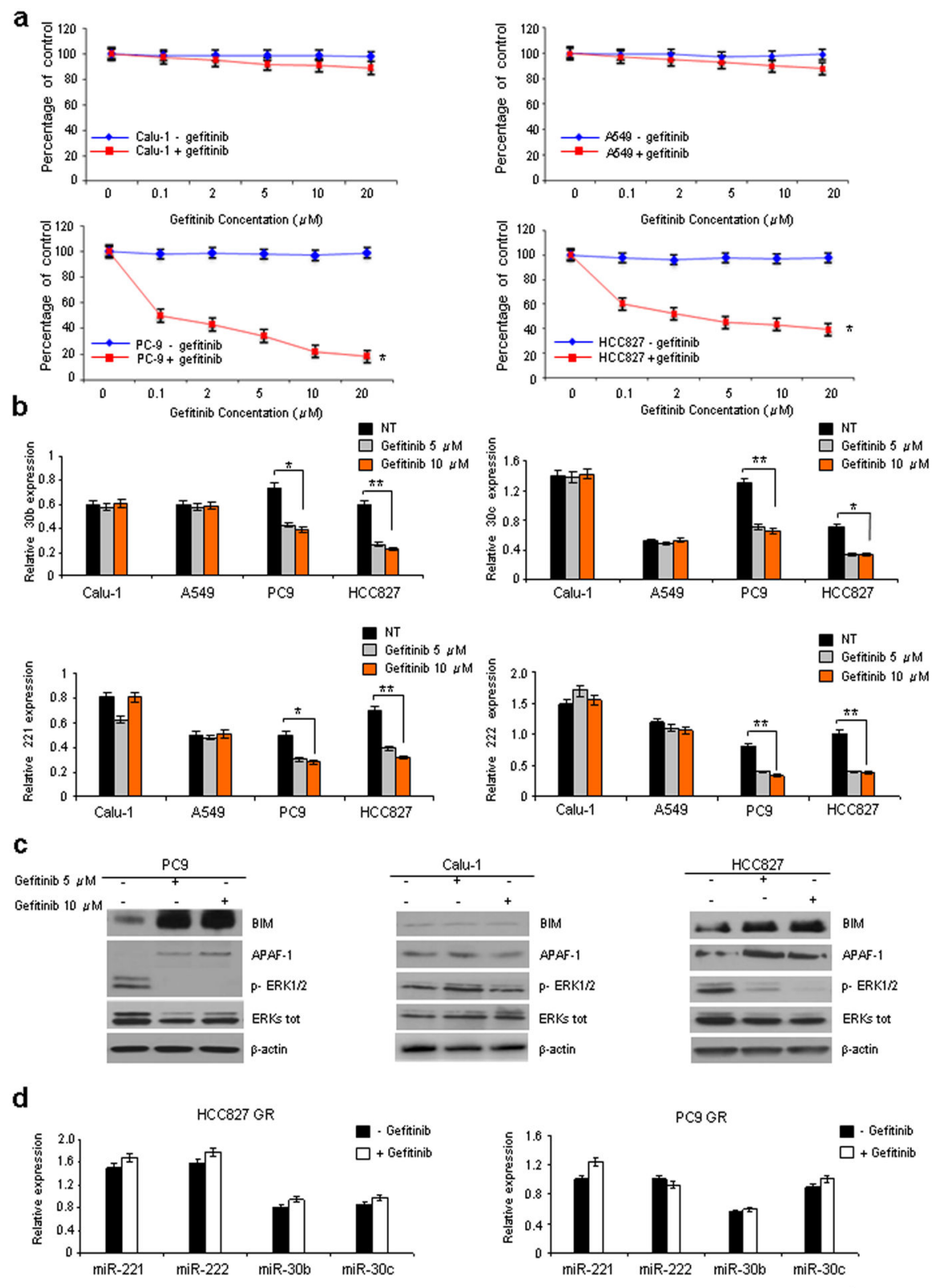

Figure 3. Gefitinib downregulates miR-221-222 and 30b-c

(a) Calu-1, A549, PC9 and Hcc827 cells were treated with increasing concentrations of gefitinib. Cell viability, relative to untreated controls, was measured after $24 \mathrm{~h}$. Each data point represents the mean \pm s.d. of five wells. (b) qRT-PCR showing miR-30b-c and -221-222 down-regulation only in PC9 and Hcc827 gefitinib-sensitive cells and not in Calu-1 and A549-resistant cells after treatment with 5 or $10 \mu \mathrm{M}$ gefitinib. (c) PC9, Calu-1 and Hcc827 cells were treated for $24 \mathrm{~h}$ with 5 or $10 \mu \mathrm{M}$ gefitinib. An increase of BIM and APAF-1 expression and a decrease of ERKs phosphorylation were observed only in the Hcc827 and PC9 gefitinib-sensitive cells but not in the Calu- 1 -resistant cells. $\beta$-actin was used as loading control. (d) qRT-PCR showing that miR-30b-c and -221-222 expression did not decrease in PC9 GR and HCC827GR cells (with acquired gefitinib resistance) exposed 
to $10 \mu \mathrm{M}$ gefitinib for $24 \mathrm{~h}$. All quantitative data were generated from a minimum of three replicates. Error bars depict \pm s.d. Two tailed student's $t$ test was used to determine $P$ values. $* P<0.001, * * P<0.05$. 
a
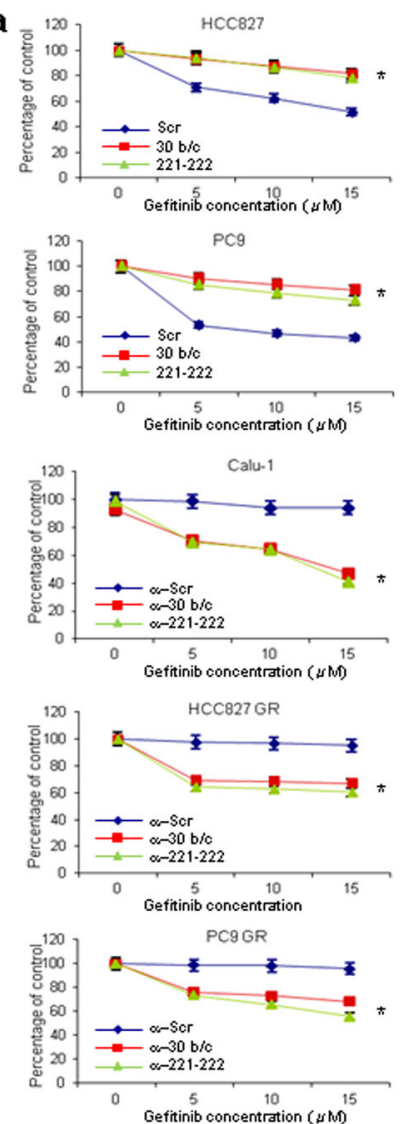
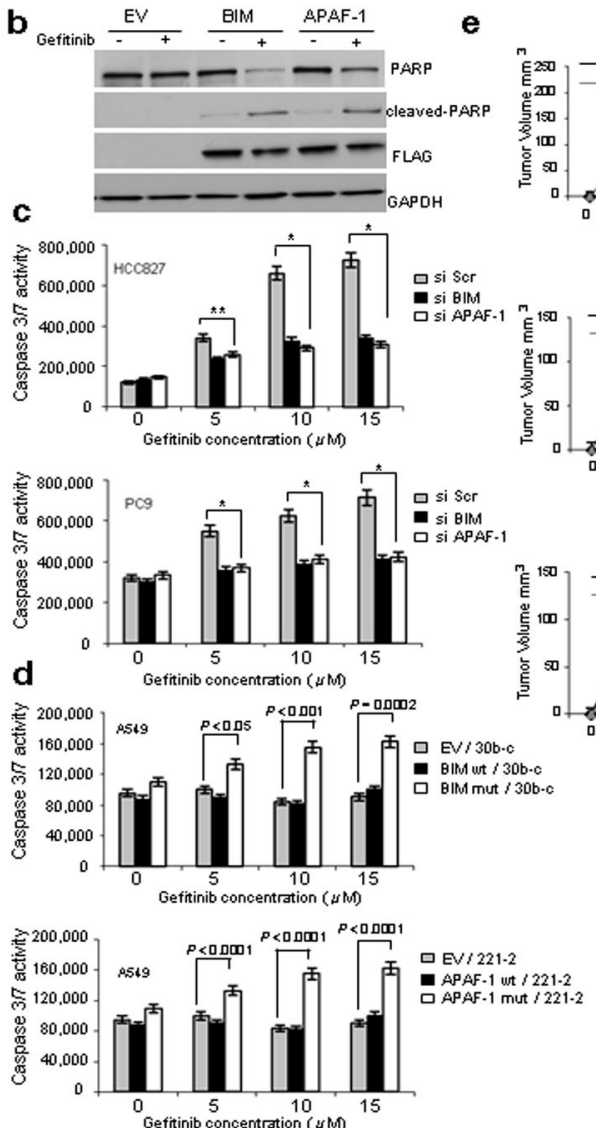
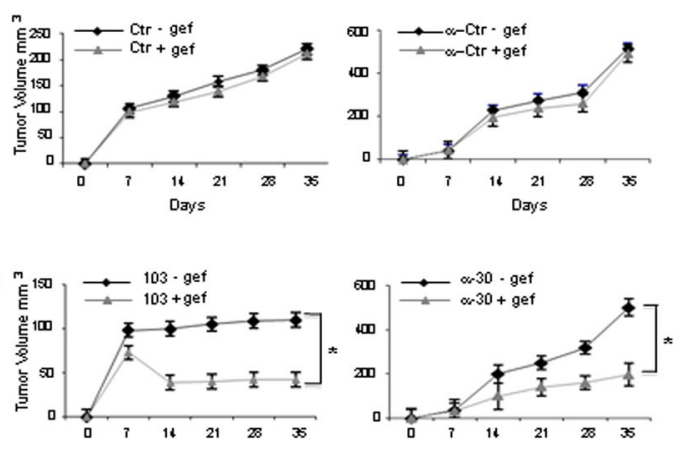

Days

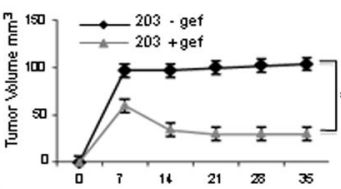

Days

$\mathbf{f}$

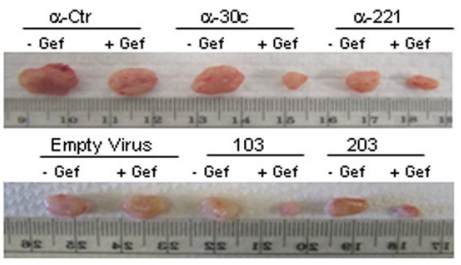

Figure 4. MiR-30b-c, 221-222, 103, 203 regulates gefitinib sensitivity

(a) Parental and resistant HCC827 GR and PC9GR and Calu-1 cells treated with increasing concentrations of gefitinib. Each data point represents the mean \pm s.d of 6 wells. (b) Western blot showing an increase in PARP cleaved fragment after BIM and APAF-1 over-expression and gefitinib treatment $(15 \mu \mathrm{M})$ in A549 cells. (c) BIM and APAF-1 silencing in HCC827 and PC9 cells reduces the response to gefitinib. (d) Overexpression of miR-30b/c- and -221/222-insensitive BIM and APAF-1 cDNAs, induces gefitinib sensitivity in A549 cells. (e-f) MiR-103, -203 overexpression and miR-30c, -222 silencing increase gefitinib sensitivity in vivo. (e) Growth curve of engrafted tumors and (f) comparison of engrafted tumors in nude mice injected with A549 cells stable infected with anti-miR-control (ctr), anti-30c, anti-221 and with miR-103 and -203 or an empty virus as control. The images show average-sized tumors from among five of each category. In $\mathbf{a}, \mathbf{c}, \mathbf{d}$ and $\mathbf{e}$ error bars indicate \pm s.d. $* P<0.001,{ }^{* *} P<0.05$ by two tailed student's $t$ test. 
a
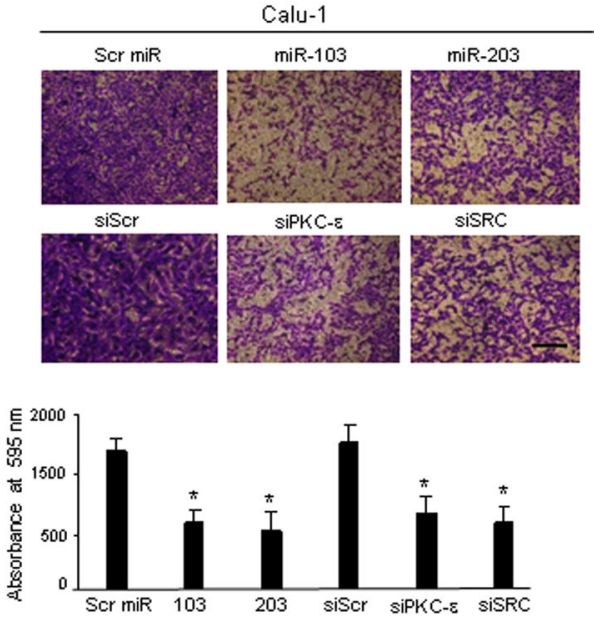

b
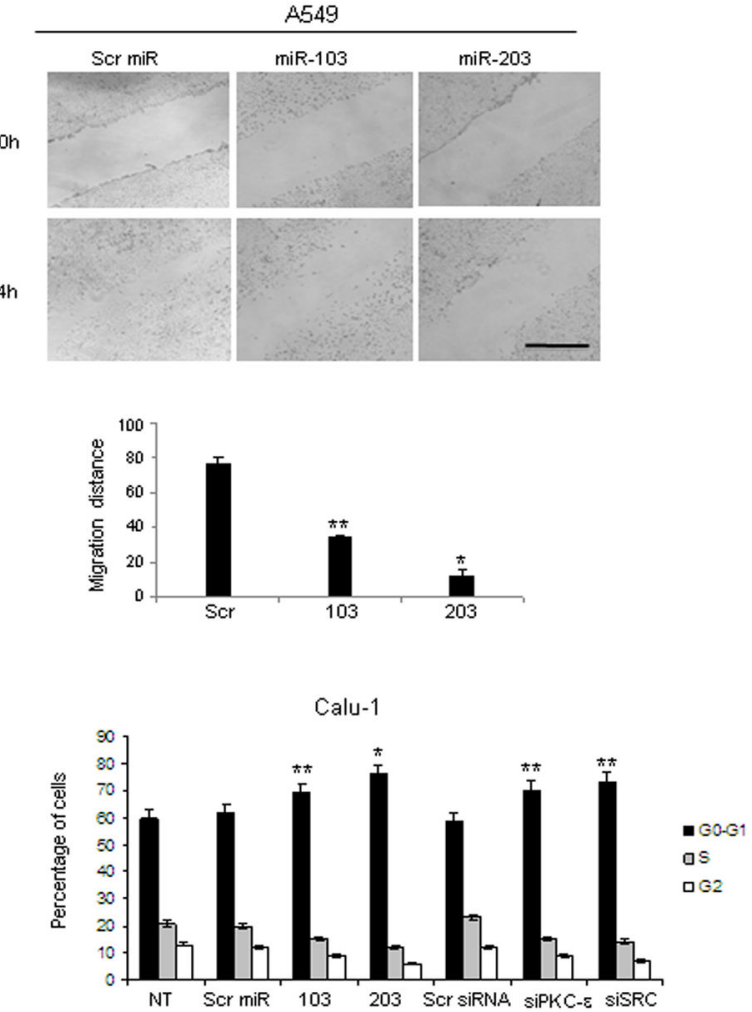

c

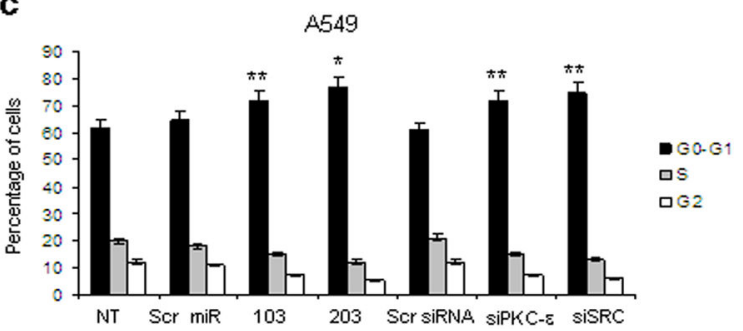

Figure 5. MiR-103, 203 inhibit migration and proliferation of NSCLC

(a)Representative pictures of cells that migrated through the filter and stained with crystal violet. Scale bar, $40 \mu \mathrm{m}$. The results are means \pm s.d, $n=3$. ${ }^{*} P<0.001$. (b) Representative photographs of scratched areas of confluent monolayer of A549 cells transfected with $\mathrm{miR}-103,-203$ or ctr miR at $0 \mathrm{~h}$ and $24 \mathrm{~h}$ after wounding with a pipet tip. Scale bar, $500 \mu \mathrm{m}$. Significance values of $* P<0.00001$ and $* * P<0.001$ relative to miR scrambled transfected cells. (c) Flow cytometric distributions of Calu-1 and A549 cells transfected with control miR, miR-103, miR-203, control siRNA, PKC- $\varepsilon$ and SRC siRNAs. The effect of miR-203 on cell cycle is slightly stronger than that of miR-103, as assessed by the ratio between GoG1 and S phases. (A549 G0-G1/S NT=3.1 \pm 0.2 ; $\mathrm{Src} \mathrm{miR}=3.6 \pm 0.2 ; 103=4.8 \pm 0.25 ; 203=$ $6.4 \pm 0.2$; scr siRNA $=2.9 \pm 0.3$; siPKC- $\varepsilon=4.8 \pm 0.1 ;$ siSrc $=5.7 \pm 0.25$. Calu- 1 G0-G1/S NT= $2.8 \pm 0.34$; Scr $\mathrm{miR}=3.1 \pm 0.21 ; 103=4.3 \pm 0.25 ; 203=6.3 \pm 0.25$; Scr siRNA= 2.5 \pm 0.21 ; siPKC- $\varepsilon=4.6 \pm 0.26 ; \operatorname{siSrc}=5.2 \pm 0.32$ ). All quantitative values show mean $\pm \mathrm{s} . \mathrm{d}, n=5$. Two tailed student's $t$ test was used to determine $P$ values between $\mathrm{G} 0-\mathrm{G} 1 / \mathrm{S}$ ratio. $* P<0.00001$ versus scr miR. $* * P<0.005$ versus scr miR. 
a

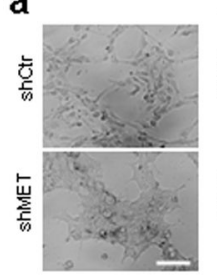

b

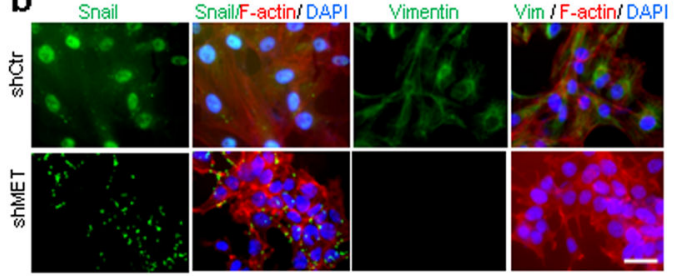

d
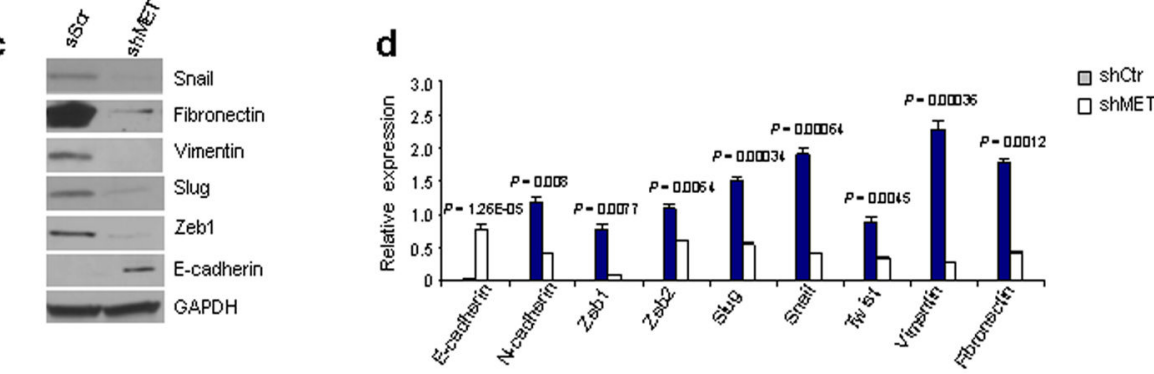

e

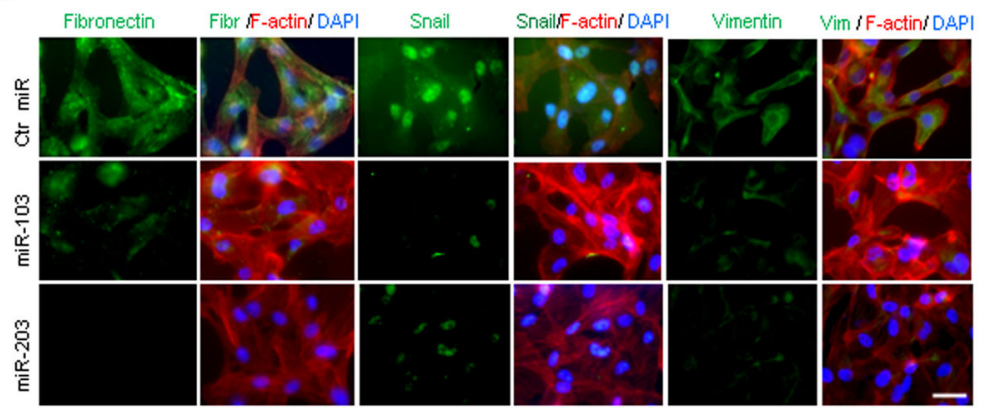

f

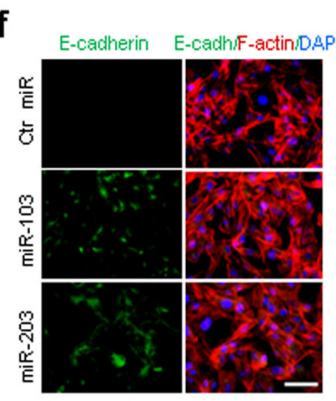

g

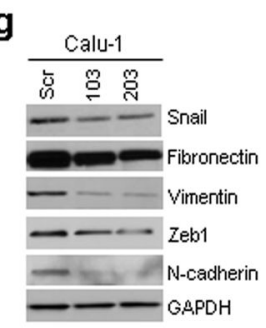

h

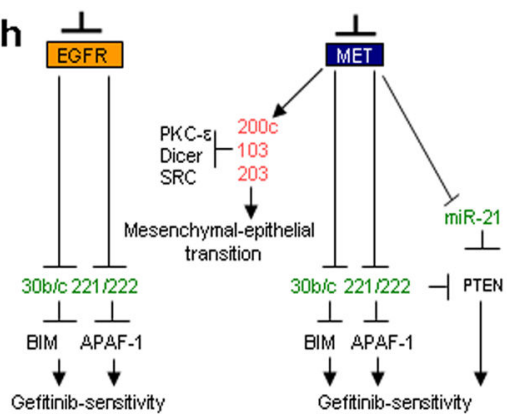

Figure 6. c-Met knockdown induces MET through miR-103 and -203

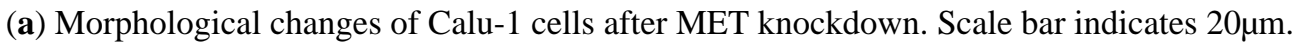

(b) Immunofluorescence: Snail, mesenchymal intermediary filament vimentin and Ncadherin in Calu-1 shControl and Calu-1 shMET. Snail expression is strong and nuclear in Calu-1 ShCtr and is weaker and cytoplasmic in Calu-1 ShMET. Scale bar, 20 $\mu \mathrm{m}$. (c) Western blots showing the downregulation of mesenchymal proteins as fibronectin, vimentin and Snail and the upregulation of E-cadherin after MET knockdown in Calu-1 cells. Loading control, GAPDH. (d) qRT-PCR showing the expression of epithelial and mesenchymal markers in Calu-1 ShCtr and Calu-1 shMET. (e) Immunofluorescence: fibronectin, Snail and vimentin expression decreases after miR-103 or -203 overexpression

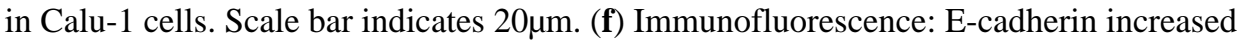


signal after miR-103 or -203 enforced expression in Calu-1 cells. Scale bar, 40 $\mu \mathrm{m}$. (g) Immunoblot showing the down-regulation of mesenchymal markers after miR-103 or -203 overexpression. (h) A model is reported in which MET inhibition elicits upregulation of miR-103 and -203, which in turn, downregulating PKC-E, Dicer and SRC, induce gefitinibsensitivity and mesenchymal-epithelial transition (MET). MET knockdown induces also miR-30b/c, -221/222 and -21 downregulation and consequent gefitinib-sensitivity through BIM, APAF-1 and PTEN upregulation. EGFR knockdown decreases miR-221/222 and $-30 \mathrm{~b} / \mathrm{c}$ expression levels. In red are the up-regulated and in green the down-regulated miRs after EGFR and MET silencing. Results are representative of at least four independent experiments. $P$ values were obtained by two-tailed student's $t$ test. Error bars represent standard deviation. 